RESEARCH ARTICLE SUMMARY

NEURODEVELOPMENT

\title{
Temporal patterning of apical progenitors and their daughter neurons in the developing neocortex
}

\author{
L. Telley* ${ }^{*}$, G. Agirman $\dagger$, J. Prados, N. Amberg, S. Fièvre, P. Oberst, G. Bartolini, \\ I. Vitali, C. Cadilhac, S. Hippenmeyer, L. Nguyen, A. Dayer, D. Jabaudon*
}

INTRODUCTION: The cerebral cortex is a cellularly heterogeneous structure whose neuronal circuits underlie high-order cognitive and sensorimotor information processing. Neocortical neuronal diversity emerges from interactions between intrinsic genetic programs and environment-derived signals, but how these processes unfold and interact in the developing brain is still unclear. During embryogenesis, distinct subtypes of glutamatergic neurons are sequentially born and differentiate from progenitors located in the ventricular zone below the cortex. The aggregate neurogenic competence of these ventricular zone progenitors (i.e., apical progenitors) hence progresses as corticogenesis proceeds, and temporal molecular patterning is thought to be a driving force for this process. Such temporal patterning is an evolutionarily conserved strategy to generate neuronal diversity, but in contrast to Drosophila, in which key molecules of tempo- ral specification have been identified, how this occurs in mammals remains poorly understood.

RATIONALE: Although the cell type diversity of the adult neocortex is increasingly well characterized, how pre- and postmitotic developmental molecular programs unfold and interact during development remains unknown. This in part reflects our limited ability to assess molecular states at defined time points and in specific cell types with high temporal resolution. Here, we overcame this obstacle by combining FlashTag, a method to pulse-label isochronic cohorts of ventricular zone-born neurons and their mother cells, with singlecell RNA sequencing. Using this approach, we traced the transcriptional trajectories of successive generations of apical progenitors and their respective daughter neurons in mice from embryonic day 12 to day 15 , as layer 6,5 , 4, and $2 / 3$ neurons are successively generated.

\author{
Temporal molecular \\ patterning in the mouse \\ neocortex. Evolutionarily con- \\ served, temporally patterned \\ genes drive apical progenitors \\ from internally directed to \\ exteroceptive states. The PRC2 \\ complex epigenetically drives \\ this progression (not shown). \\ Embryonic age-dependent \\ molecular marks are \\ transmitted from apical \\ progenitors to newborn \\ neurons as successive ground \\ states, onto which essentially \\ conserved differentiation \\ programs are applied, \\ together with later-occurring \\ environment-dependent signals \\ Thus, temporal molecular birth- \\ marks in progenitors act in their \\ postmitotic progeny to seed \\ adult neuronal diversity.
}

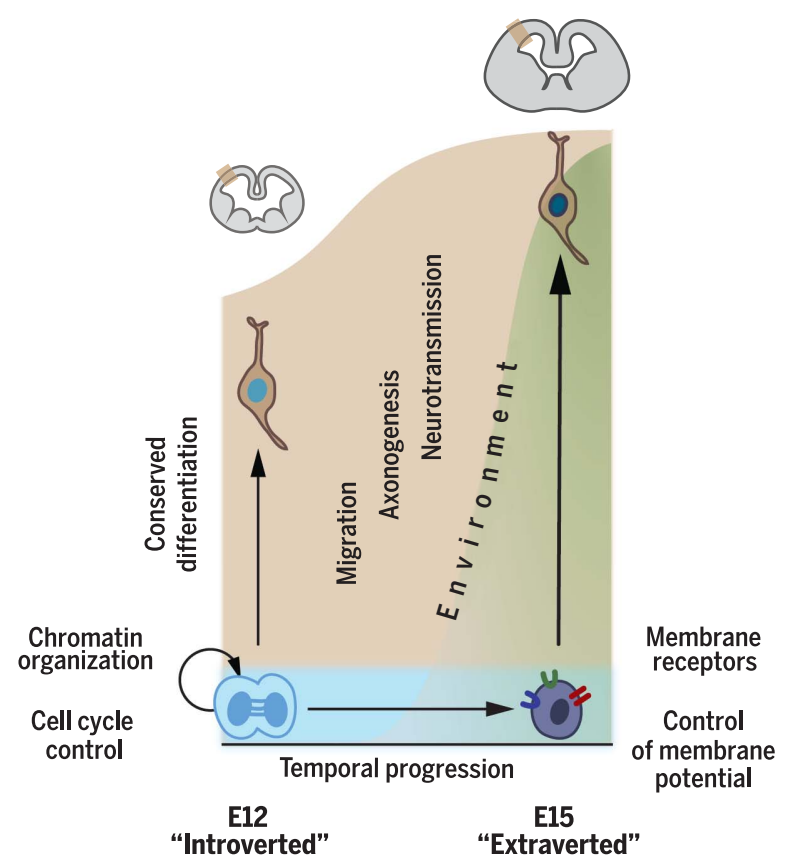

RESULTS: Using temporal analysis of apical progenitor molecular states, we identified a core set of evolutionarily conserved, temporally patterned genes that sequentially unfold during development. These dynamically expressed genes drive apical progenitors from internally directed ("introverted") to more exteroceptive ("extraverted") states. Initially, cell cycle-related and nucleus/ chromatin-related processes are prominent ac-

ON OUR WEBSITE

Read the full article at http://dx.doi. org/10.1126/ science.aav2522 related proteins, and excitability-related proteins. Temporally patterned genes are transmitted from apical progenitors to their neuronal progeny as successive ground states, onto which essentially conserved early postmitotic differentiation programs are applied. This process is evolutionarily conserved, because in human embryos, the transcriptional dynamics of the orthologs of this gene set are recapitulated in apical progenitors and their daughter neurons.

Whereas acquisition of ground states by daughter neurons is largely environmentindependent, cell-extrinsic processes come into play at later stages to sculpt final identity, as demonstrated by the progressive loss of apical progenitor-derived molecular birthmarks and the emergence of input-dependent transcriptional programs. This was particularly striking for differentiating layer 4 neurons, whose postnatal development strongly depends on synaptic input, suggesting that environmental sensibility may be prefigured prior to sensory experience. Finally, using a loss-of-function approach, we demonstrate that the temporal progression in apical progenitor states is epigenetically regulated by the Polycomb repressor complex PRC2, such that loss of PRC2 leads to an acceleration of apical progenitor neurogenic competence, as revealed by precocious generation of normally later-born cell types.

CONCLUSION: Our work provides a functional account of molecular programs at play in apical progenitors and their daughter neurons during corticogenesis in mice, and reveals that epigenetically regulated temporal molecular birthmarks in apical progenitors act in their postmitotic progeny as seeds for neuronal diversity. Conserved differentiation programs, together with later-occurring environment-dependent signals, then act on these sequential ground states to drive newborn neurons toward their final, cell type-specific identities. ․

The list of author affiliations is available in the full article online. *Corresponding author. Email: ludovic.telley@unil.ch (L.T.); denis.jabaudon@unige.ch (D.J.)

†These authors contributed equally to this work.

Cite this article as L. Telley et al., Science 364, eaav2522

(2019). DOI: 10.1126/science.aav2522 
RESEARCH ARTICLE

NEURODEVELOPMENT

\section{Temporal patterning of apical progenitors and their daughter neurons in the developing neocortex}

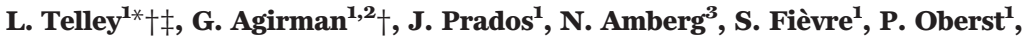 \\ G. Bartolini' ${ }^{1}$, I. Vitali ${ }^{1}$, C. Cadilhac', S. Hippenmeyer ${ }^{3}$, L. Nguyen ${ }^{2}$, \\ A. Dayer ${ }^{1,4}$, D. Jabaudon ${ }^{1,5 *}$
}

During corticogenesis, distinct subtypes of neurons are sequentially born from ventricular zone progenitors. How these cells are molecularly temporally patterned is poorly understood. We used single-cell RNA sequencing at high temporal resolution to trace the lineage of the molecular identities of successive generations of apical progenitors (APs) and their daughter neurons in mouse embryos. We identified a core set of evolutionarily conserved, temporally patterned genes that drive APs from internally driven to more exteroceptive states. We found that the Polycomb repressor complex 2 (PRC2) epigenetically regulates AP temporal progression. Embryonic age-dependent AP molecular states are transmitted to their progeny as successive ground states, onto which essentially conserved early postmitotic differentiation programs are applied, and are complemented by later-occurring environment-dependent signals. Thus, epigenetically regulated temporal molecular birthmarks present in progenitors act in their postmitotic progeny to seed adult neuronal diversity.

A dult neuronal diversity emerges from the interaction between progenitor-derived genetic information and environment-derived signals, but how these processes unfold and interact in the developing brain is still unclear. In the neocortex, whose neurons are required for high-order cognitive and sensorimotor information processing in mammals, the temporal molecular patterning of progenitors is thought to be a driving force for their progression in neurogenic competence (1-3). Such temporal patterning is an evolutionarily conserved strategy to generate neuronal diversity, but in contrast to Drosophila, in which key molecules of temporal specification have been identified (4), the manner in which this occurs in mammals remains poorly understood. Although the singlecell diversity of the neocortex is increasingly well characterized (5-11), it remains unknown how temporally progressing pre- and postmitotic programs in APs and neurons interact to give rise to the neuronal diversity of the adult neocortex. One reason for this has been the lack of a technique allowing the precise dissection of temporal

\footnotetext{
${ }^{1}$ Department of Basic Neurosciences, University of Geneva, Geneva, Switzerland. ${ }^{2}$ GIGA-Stem Cells, University of Liège, C.H.U. Sart Tilman, Liège, Belgium. ${ }^{3}$ Institute of Science and Technology Austria, Klosterneuburg, Austria. ${ }^{4}$ Department of Psychiatry, Geneva University Hospital, Geneva, Switzerland. ${ }^{5}$ Clinic of Neurology, Geneva University Hospital, Geneva, Switzerland.

*Corresponding author. Email: ludovic.telley@unil.ch (L.T.); denis. jabaudon@unige.ch (D.J.) †These authors contributed equally to this work. łPresent address: Department of Basic Neuroscience, University of Lausanne, Lausanne, Switzerland.
}

transcriptional states in specific cell types. Here, we overcame this limitation by using FlashTag (FT), a high-temporal resolution method to pulselabel APs and their daughter neurons $(12,13)$ and trace the transcriptional trajectories of successive generations of progenitors and isochronic cohorts of their daughter neurons from embryonic day (E) 12 to E15, as layer (L) 6, L5, L4, and L2/3 neurons are successively generated (3).

\section{Identifying dynamic transcriptional states in APs and neurons}

After microdissection of the putative somatosensory cortex, we used fluorescence-activated cell sorting (FACS) to collect $\mathrm{FT}^{+}$cells after 1 hour, as APs are still dividing; after 24 hours, as daughter cells are transiting through the subventricular zone; or after 96 hours, once daughter neurons have entered the cortical plate (Fig. $1 \mathrm{~A}$ and fig. S1, A to C). We performed single-cell RNA sequencing at each of these three differentiation stages at four embryonic ages (E12, E13, E14, and E15), which yielded a total of 2756 quality-controlled cells across 12 conditions for analysis (fig. S1, C and D, and supplementary materials).

Analysis of cellular transcriptional identities by t-distributed stochastic neighbor embedding (t-SNE) dimensionality reduction revealed that cells were organized on the basis of collection time (i.e., differentiation status): 1-hour-old, 1-dayold, and 4-day-old cells formed three main groups of cells that corresponded essentially to (i) APs, (ii) basal progenitors (BPs) and 1-day-old neurons (N1d), and (iii) 4-day-old neurons (N4d), as indicated by the combined expression of cell type-specific markers (Fig. 1B) (12). At each of these differentiation stages, cells born at successive embryonic ages tended to cluster together, forming chronotopic maps, which were particularly apparent for APs and 1-day-old daughter cells but were less striking, although still discernible, in 4-day-old neurons. This suggests that birthdaterelated transcriptional features are strong determinants of AP and of initial neuron identity, but also that non-birthdate-related programs are implemented during differentiation.

Together, these data reveal two axes of transcriptional organization: (i) a birthdate axis, corresponding to the temporal progression in $\mathrm{AP}$ transcriptional states at sequential embryonic ages, and which also emerged from unbiased analysis of single-cell trajectories (fig. S1E) (14); and (ii) a differentiation axis, corresponding to the birth and maturation of their daughter neurons. These two cardinal processes are thus the major source of transcriptional diversity in the developing neocortex.

We used a graph-based cluster analysis to investigate the diversity of differentiation stageand birthdate-specific cells (Fig. 1C) and differential expression analysis to identify type-enriched transcripts, whose temporal patterns of expression were confirmed by in situ hybridization (Fig. 1, D and E; figs. S2 and S3; and data S1; see also http://genebrowser.unige.ch/telagirdon/). Cluster analysis identified four embryonic agedefined AP transcriptional states, as well as two embryonic age-defined basal progenitor populations also previously reported (15) (Fig. 1C). Two classes of 1-day-old neurons could be distinguished, early-born cells (i.e., E12 or E13-born) and later-born cells (i.e., E14 or E15-born). These two classes of neurons displayed early-onset expression of deep- and superficial-layer genes, which foreshadowed their upcoming laminarelated identity. Early on, however, classical deeplayer markers were also expressed by late-born neurons (fig. S4A), as previously reported $(16,17)$. By 4 days of age, however, neurons with mutually exclusive expression of classical laminaspecific markers such as Bcl11b (a L5/deep layer marker), Rorb (L4), and Pou3f2 (L2/3) emerged (Fig. 1C, right, and fig. S4, A and B). Thus, lateborn neurons initially transiently display some molecular features of earlier-born neurons, which they repress as they undergo fate refinement.

Of note, in addition to these ventricular zone (VZ)-born cell populations, two types of non-VZborn cells were detected. First, GABAergic interneurons were primarily detected 4 days after FT labeling, consistent with migration into the dorsal pallium after FT labeling of their progenitors in the ventral pallium $(18,19)$ (Fig. 1C and fig. S4C). A small fraction of these interneurons (16\%) was detected 1 hour after FT labeling, especially at late embryonic stages, supporting reports of inhibitory interneurons migrating radially to contact the ventricle in the dorsal pallium late in corticogenesis $(20,21)$. Second, astrocytes were exclusively detected 4 days after E15 FT labeling (i.e., at P0); these likely correspond to ventral 

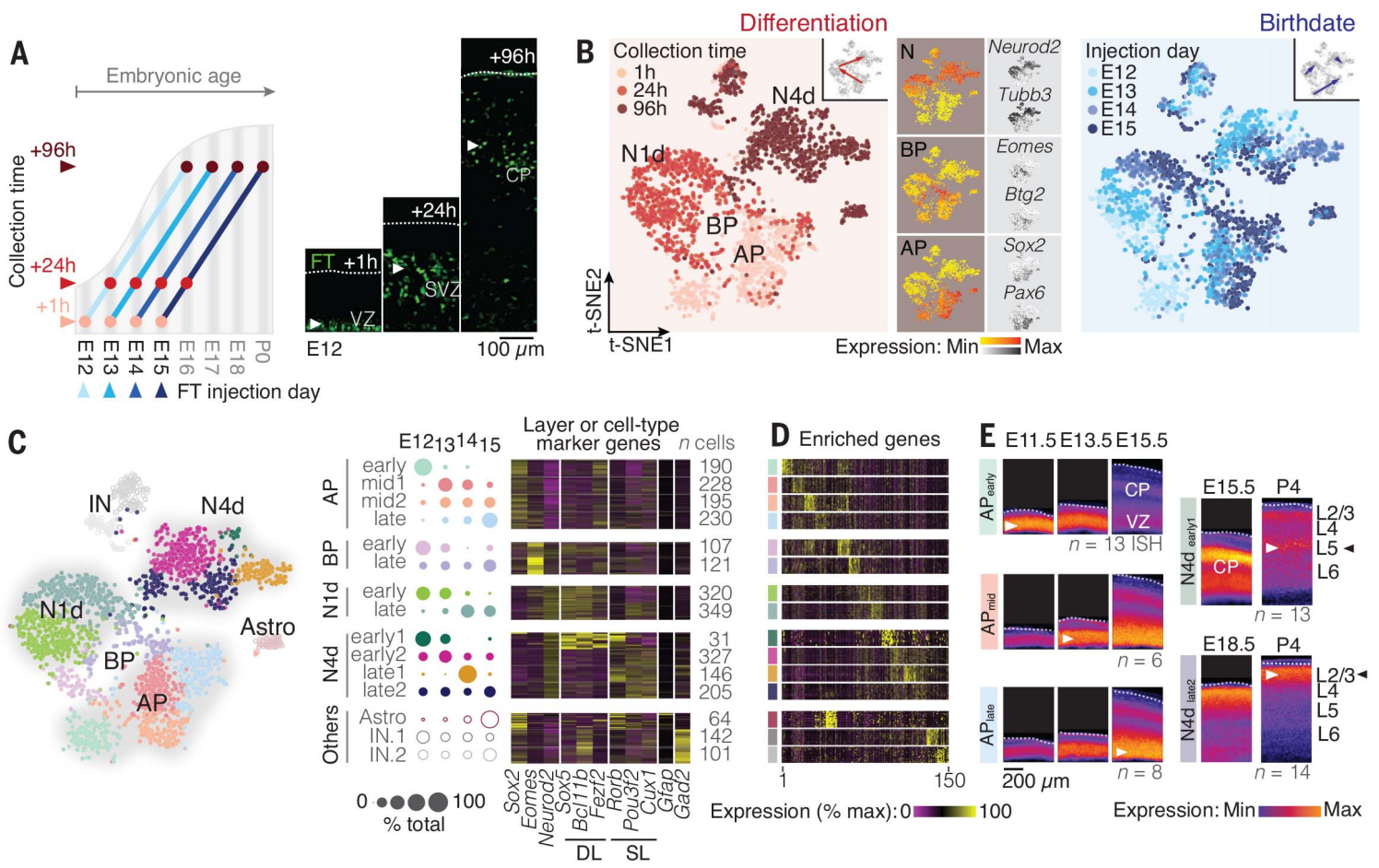

Fig. 1. Birthdate- and differentiation stage-related cellular diversity in the developing neocortex. (A) Schematic illustration of the experimental procedure. M-phase APs were labeled by FT injection performed at E12, E13, E14, or E15, and isochronic cohorts of APs and daughter cells were collected 1, 24, or 96 hours later. (B) t-SNE representation of the single-cell RNA sequencing dataset revealing the transcriptional organization of the cells according to the time at which they were collected (i.e., their differentiation status) and the day on which the injection was performed (i.e., their birthdate). Apical progenitors, basal progenitors, and neurons can be distinguished by their combinatorial expression of key marker genes ( $n=20$ transcripts).

(C) Cluster analysis reveals transcriptionally distinct and temporally dynamic cellular clusters. Cluster nomenclature reflects prevalence of the cluster at a given embryonic age (early, E12/E13; late, E14/E15). Cells in these clusters express classical layer and cell type marker genes in accordance with their birthdate and differentiation status. (D) Expression of the top 10 most enriched genes per cluster highlights cellular diversity. See also data S1. (E) Spatiotemporal expression of cluster-specific transcripts with in situ hybridization (ISH) from the Allen Developing Mouse Brain Atlas. Color-coded images represent the average expression for representative transcripts (see also figs. S2 and S3). AP, apical progenitors; Astro, astrocytes; BP, basal progenitors; CP, cortical plate; DL, deep layer; IN, interneurons; N, neurons; N1d, 1-day-old neurons; N4d, 4-day-old neurons; SL, superficial layer; SVZ, subventricular zone. pallium-born astrocytes migrating into the cortex $(22,23)$ (Fig. 1C and fig. S4D). These two cell types were not further investigated in this study. Together, these results indicate that APs transit through temporally dynamic transcriptional states during corticogenesis as daughter neurons progressively acquire cell type-specific transcriptional features.

\section{APs progress from "introverted" to "extraverted" transcriptional states}

We used two axes of investigation to address the transcriptional dynamics of differentiating neurons and of APs: (i) the transcriptional differentiation of neurons born on each embryonic day between E12 and E15 (Fig. 2, A to C) and (ii) the progression in AP transcriptional states during this time period (Fig. 2, D to F).

We first examined the transcriptional programs expressed by differentiating neurons born at each embryonic age (Fig. 2, A to C). For this purpose, we used an unsupervised approach in which single cells were unbiasedly ordered on a linear path on the basis of their transcriptional profile (24). This pseudo-time (i.e., pseudodifferentiation) alignment approach outlined successive transcriptional waves driving differentiation (12) (Fig. 2A, fig. S5, and data S2). Remarkably, the sequential unfolding of gene expression was essentially conserved across embryonic ages, as revealed by closely matching gene expression dynamics (Fig. 2B and fig. S5, A to E). Constant gene expression did not simply reflect the constant activity of a small number of "pan-neuronal" genes (e.g., Neurod2) but instead reflected tightly overlapping differentiation programs, because more than half of the expressed genes had highly correlated expression dynamics ( $R>0.7$; fig. S5C). Accordingly, gene ontologies were conserved across embryonic ages (Fig. 2C, fig. S5F, and data S3). Thus, neuronal differentiation programs appear largely conserved across corticogenesis.

We next addressed the temporal progression in AP transcriptional states, using the approach described above. This pseudo-time alignment approach appropriately ordered E12-, E13-, E14-, and E15-dividing APs, effectively constituting a pseudo-birthdate axis (Fig. 2D). This allowed us to determine individual gene expression dynamics in APs across corticogenesis, which were reflected by in situ hybridization temporal series
(Fig. 2E; fig. S6, A to C; and data S4). Clustering of transcripts based on their expression dynamics showed how transcriptional programs unfold in APs (Fig. 2, E and F, and data S4 and S5). Initially (E12, E13), typically cell-intrinsic ("introverted") programs were at play: Transcripts coding for nuclear proteins (e.g., RpaI, involved in DNA replication or the Top $2 A$ DNA topoisomerase) and cell cycle regulators (e.g., Ccne1, E2f1, E2f8) were increased, consistent with active cell cycle regulation processes. Similarly, transcripts involved in the regulation of gene expression and chromatin structure were prominent [e.g., Hmga2, which promotes self-renewal of neural progenitors (25), and Trim27, which interacts with the Polycomb complex to repress gene expression (26)]. Finally, cell death modulators (e.g., Siva1, Scrib) were also actively transcribed, suggesting some level of control over progenitor pool size (27).

Later on (E14, E15), exteroceptive ("extraverted"), environment sensing-related programs predominated (Fig. 2F). This included ion transport-related processes, in line with a bioelectrically controlled progression of AP competence (28) and consistent with the increased frequency of calcium waves in APs later in corticogenesis (29). Similarly, cell-cell 
Fig. 2. Neuronal differentiation programs are conserved while APs shift from internally directed to exteroceptive transcriptional states. (A) Principal components analysis (PCA) showing spontaneous organization of cells along a differentiation axis (i.e., from AP to N1d to N4d). Black line indicates pseudo-differentiation axis onto which cells were aligned. (B) Clustering of gene expression kinetics reveals sequential transcriptional waves. (C) Examples of gene ontology processes associated with each of the expression waves, along with sample corresponding genes. Note conserved dynamics across embryonic ages. See also fig. S5 and data S2 and S3. (D) PCA of AP transcriptional identity showing chronotopic organization along a birthdate axis (i.e., from E12 to E15). (E) Cluster analysis reveals distinct dynamics of AP gene expression during corticogenesis. (F) Examples of gene ontology terms associated with each expression dynamics, along with select corresponding genes. See also fig. S6 and data S4 and S5. (G) Schematic summary of the findings. (H) APs cycle progressively more slowly as corticogenesis unfolds. ${ }^{*} P<0.05$ (Student $t$ test); n.s., not significant. (I) The glutamate transporter transcript SIcla3 (Glast) is expressed by APs late in corticogenesis. Blockade of GLAST with TBOA (35) increases extracellular glutamate at late but not early embryonic ages, as detected by activation of ionotropic glutamate receptors in patch-clamped APs. Scale bars, 5 min, 20 pA. BrdU, 5-bromo-2'-deoxyuridine; EdU, 5-ethynyl-2'-deoxyuridine; FDR, false discovery rate; PC, principal component; $\mathrm{Vm}$, membrane potential.

and cell-matrix interaction-related processes (e.g., Bcan) increased, as did lipid metabolism process (e.g., Fabp7, Acadvl), which has been linked with progenitor fate in adult neuronal stem cells (30). In line with the induction of exteroceptionrelated programs, late APs also expressed genes typically associated with neuron-related processes such as synaptogenesis and neurotransmission, which suggests that corresponding exteroceptive programs are progressively implemented in successive generations of APs and in their postmitotic neuronal progeny. Finally, glia-related processes emerged, foreshadowing the upcoming generation of this cell type later in corticogenesis. Together, these data suggest that as corticogenesis unfolds, APs shift from mostly internally driven programs to environment-sensing ones (Fig. 2G).

We used two examples to illustrate the functional correlates of this progression in transcriptional states. First, we investigated the net effect of the dynamics of cell cycle control-related transcripts (which predominate at early stages) by measuring cell cycle duration specifically in APs $\left(\mathrm{SOX2}^{+}\right.$cells) (Fig. $\left.2 \mathrm{H}\right)$. This revealed a $\sim 50 \%$ increase in cell cycle length between E12 and E15 (from 8 hours to 12 hours), confirming and extending previous results $(31,32)$. Lengthening of the cell cycle essentially reflects a lengthening of $\mathrm{G}_{1}$, a critical phase for environment sensing and fate decision $(31,33,34)$ (fig. S6D), such that this progression in cycling dynamics is congruent
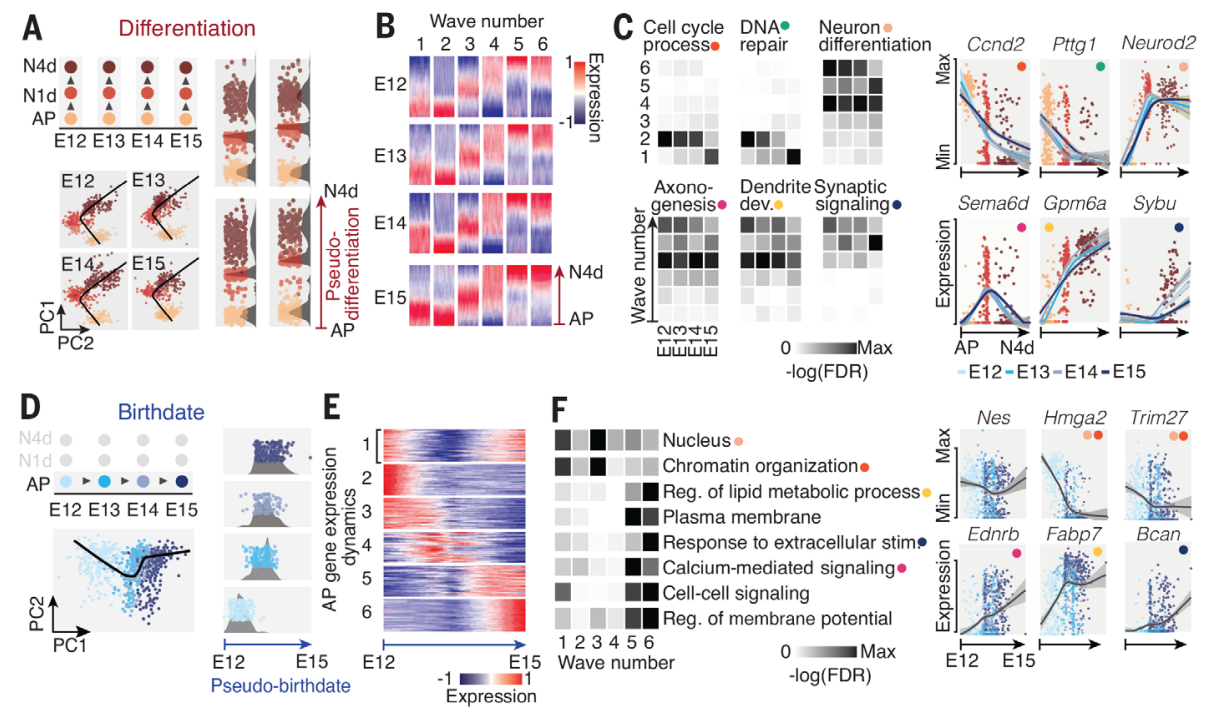

G
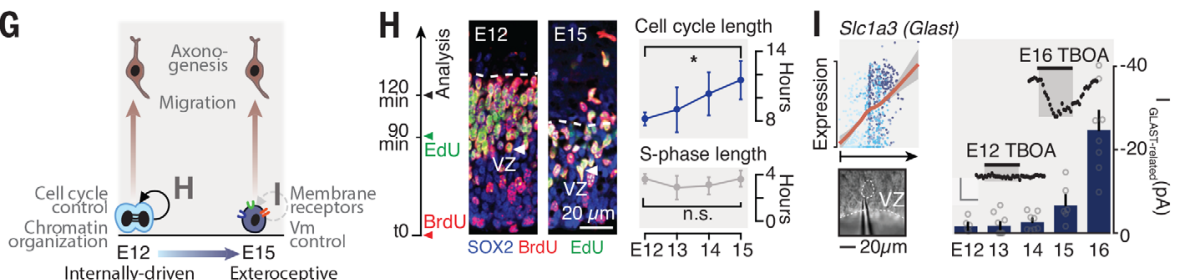

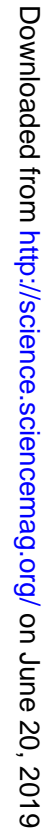

with the overall progression in AP behavior during corticogenesis.

A second example was provided by the glutamate transporter transcript Slc1a3 (Glast), whose expression increases in APs as glutamatergic neurotransmission develops in the cortical plate (Fig. 2I). To directly investigate whether increased Glast transcriptional activity was accompanied by an increase in glutamate uptake by APs, we used DL-threo- $\beta$-benzyloxyaspartic acid (TBOA) to block this electrogenic transporter at distinct embryonic stages (35) and measured evoked currents using whole-cell patch clamping of APs, identified as juxtaventricular cells (13). Pharmacological blockade of this transporter increased glutamate levels at late but not early embryonic stages, consistent with a dynamic bioelectrical control over AP properties during corticogenesis (Fig. 2I) (28). Thus, the transcriptional progression from mostly internally driven processes to environment-sensing ones is accompanied by a corresponding functional progression in AP biological properties.

\section{APs and daughter neurons share molecular temporal identities}

The differentiation programs of daughter neurons are largely conserved across embryonic ages, despite the distinct identities these daughter neurons acquire later. How, then, does neuronal diversity emerge? As reported above, the chronotopic arrangement of APs is also present in their 1-day-old progeny (Fig. 1B). This suggests that embryonic age-dependent AP transcriptional programs are transmitted to their progeny to generate successive initial neuronal identities. To investigate this possibility, we next determined how dynamic transcriptional networks emerge in single cells during corticogenesis. We used a machine learning strategy to classify cells based on (i) their birthdate and (ii) their differentiation status, which identified core sets of genes ( $n=100$ per model) sufficient to classify all cells according to these two cardinal features (Fig. 3A; fig. S7, A to C; and data S6), many of which have been previously identified as regulators of progenitor and neuronal fate (tables S1 and S2). Birthdate-associated core genes were sequentially expressed by APs and their 1- and 4-day old progeny, revealing a shared temporal patterning of mother and daughter cells (Fig. 3B, top, and fig. S7D). On the other hand, the dynamics of the differentiation gene set were conserved across embryonic ages, consistent with a consensus postmitotic differentiation program, as identified earlier (Fig. 3B, bottom, and fig. S7D). This process is an evolutionarily conserved one, because in human embryos, the transcriptional dynamics of the orthologs of this gene set and the corresponding temporal patterning of daughter neurons and their mother cells was conserved in a large dataset of cortical cells (7) (Fig. 3C). Thus, the temporal patterning process identified here constitutes a conserved strategy to generate neuronal 
diversity during corticogenesis. Consistent with the increase in neuron-related ontologies in APs noted above, expression of the neuronal differentiation gene set progressively increases in APs as corticogenesis unfolds (fig. S7, E and F); the latter cells thus become progressively "neuronized" as they give rise to successive generations of postmitotic daughter cells. Taken together, these data reveal an evolutionarily conserved specification process in which neuron type identities emerge from temporally defined transcriptional ground states present in their mother cell, onto which essentially conserved postmitotic differentiation programs are applied (Fig. 3D).

\section{Role of the environment in refining neuronal identity}

The corresponding temporal birthmarks of newborn neurons and their mother AP could in principle reflect either a lineage-related process (i.e., the vertical transmission of a transcriptional state from mother to daughter cell) or an environmental process in which both cells synchro- nously respond to progressing temporal environmental cues. To distinguish between these two possibilities, we reasoned that if environment factors were driving daughter neuron temporal identity, then the temporal patterning of postmitotic neurons would be less distinct in vitro than in vivo. We thus isolated $\mathrm{FT}^{+}$APs at E12 and E15 in vitro and examined the fate of their daughter neurons. Using combined expression of CTIP2 (officially called BCL11B, an early-born neuron marker) and BRN2 (officially called POU3F2, a late-born neuronal marker) to molecularly distinguish neurons born from E12 or E15 APs (figs. S4B and S8A), we found that, as was the case in vivo, the temporal identities of E12 and E15 APs' progenies remained sharply defined in vitro (fig. S8). Together with the conserved ability of cortical progenitors to generate successive populations of neurons in vitro $(1,36)$, these findings support a largely vertical transmission of temporal birthmarks from mother to daughter cells.

Although the environment does not appear to drive the initial identity of newborn neurons,

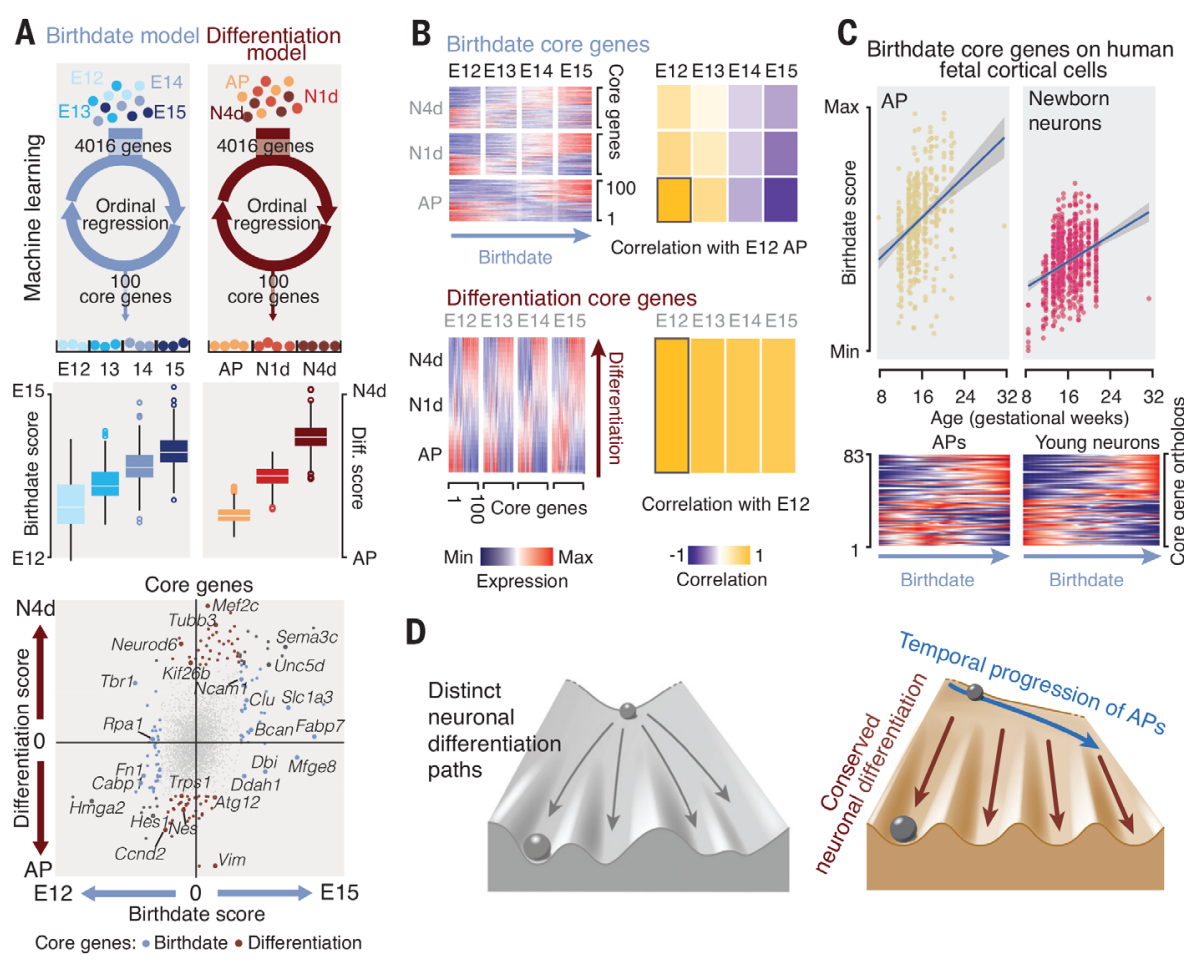

Fig. 3. Temporally progressing AP transcriptional states interact with conserved differentiation programs to generate neuronal diversity. (A) Top: Machine learning approach used to identify a core set of genes that can classify cells according to their date of birth (left) and differentiation status (right). Center: Model performance using actual dataset. Box plots show medians \pm SEM. Bottom: Weight of the core genes in predicting birthdate and differentiation status. See also tables S1 and S2 and data S6. (B) Top: Birthdate-associated core genes are temporally dynamic and daughter cells acquire embryonic stage-specific transcriptional birthmarks. See also fig. S7D. Bottom: In contrast, differentiation status-associated core genes are conserved across corticogenesis. (C) The transcriptional dynamics of the core gene orthologs (top) and the corresponding temporal patterning of daughter neurons and their mother cells (bottom) are conserved in human embryonic neocortex [dataset from (7)]. (D) Schematic representation of the findings. Left: In the classical Waddington epigenetic model, cellular diversity emerges through distinct developmental trajectories. Right: The current data show that instead, in the neocortex, developmental trajectories are conserved but initial ground states are temporally dynamic. temporal patterning fades with differentiation (see the partial loss in the chronotopic mapping in Fig. 1B), which suggests that non-birthdaterelated processes come into play as neurons mature. To examine how temporal birthmarks evolve with neuronal differentiation, we applied the pseudo-time alignment approach used above to N1d and N4d and compared it with the results obtained with APs. This revealed an overall fading of temporal patterning with differentiation, which was particularly striking for E14-born neurons. These neurons differentiate into L4 neurons, and their final identity strongly depends on environmental input (37) (Fig. 4A). Consistent with the progressive implementation of environmentdriven programs, functionally relevant inputdependent transcripts such as Nrn1 and Rorb (12, 37-39) progressively increased in E14-born neurons as they matured (Fig. 4B). Together with the temporal progression in AP exteroceptive programs, these findings suggest that the relative importance of genetic and environmental factors shifts toward the latter as neurons differentiate and corticogenesis proceeds (Fig. 4C).

\section{Mapping dynamic transcriptional landscapes during corticogenesis}

We combined the two aforementioned models to identify birthdate- and differentiation stagerelated patterns of gene expression. On the basis of the combined expression of the core genes of the two models, each cell was assigned a birthdate score and differentiation score. Cells were then embedded within a two-dimensional matrix, allowing the display of gene expression profiles as chronotypic transcriptional maps (Fig. 5A) (7). This approach revealed a variety of dynamically regulated transcriptional patterns, including within single families of genes (Fig. 5B, fig. S9, and http://genebrowser.unige.ch/telagirdon/). To identify archetypical features of gene expression, we performed a t-SNE-based cluster analysis of all transcriptional maps, revealing canonical clusters of genes with similar expression dynamics (Fig. 5C and data S7). Genes within each of these canonical clusters shared common functions, and the distinct clusters were functionally specialized (Fig. 5D, fig. S10, and data S8). This finding suggests that these transcriptional clusters represent functional units orchestrating the progression of temporal patterning during corticogenesis.

To illustrate the functional relevance of these processes in the temporal patterning of $\mathrm{AP}$ and neuronal identity, we examined the role of chromatin organization activity, which predominates in early APs (Figs. 2F and 5D), as a proof-ofprinciple process. Expression of Polycomb repressive complex 2 (PRC2), which regulates histone methylation and hence chromatin accessibility (40), provided a point of entry: The main core subunits of the complex were coexpressed in APs early in corticogenesis, and the H3K27me3 (trimethylated histone $\mathrm{H} 3 \mathrm{Lys}^{27}$ ) signature mark of PRC2 had corresponding dynamics, demonstrating temporally gated functional activity (Fig. 6A). To directly address the role of this complex in the 
Fig. 4. Temporal birthmarks fade with differentiation as environment-dependent programs are implemented.

(A) Pseudo-time alignment of APs, N1d, and N4d using the birthdate core genes shown in Fig. 3 reveals an overall fading of temporal birthmarks as neurons differentiate, particularly affecting E14-born, L4-fated neurons. (B) Environmentdependent genes from (37) are progressively up-regulated in differentiating $L 4$ neurons.

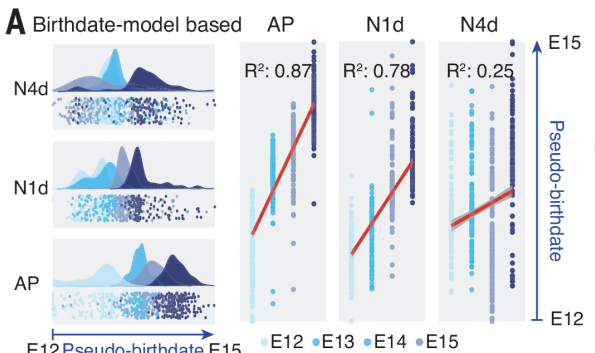

B

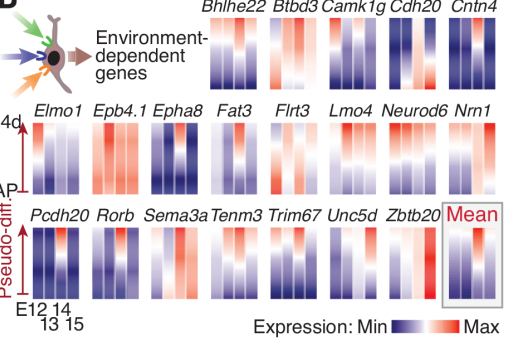

C

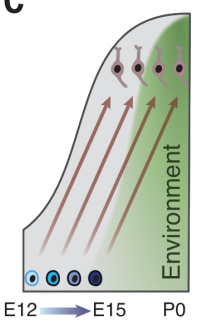

Fig. 5. Dynamic transcriptional mapping of corticogenesis.

(A) Cellular map in which cells are displayed according to their combined expression of the core genes of the birthdate and differentiation status presented in Fig. 3. Dynamic expression of genes ("transcriptional maps") throughout corticogenesis can be determined on the basis of this template (as exemplified here by Sox2 and Neurod2). (B) Example of transcriptional landscapes for select genes and corresponding validation using single-molecule fluorescent in situ hybridization (smFISH). See also fig. S9, A to C. (C) Canonical transcriptional maps can be identified by t-SNE clustering of the maps of individual genes. See also data S7. See http:// genebrowser.unige.ch/telagirdon/ for transcriptional maps for all expressed genes. (D) Genes belonging to each of the clusters have converging and specialized ontologies. See also data S8.

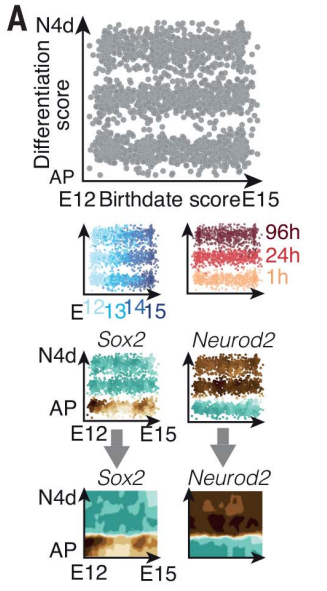

B

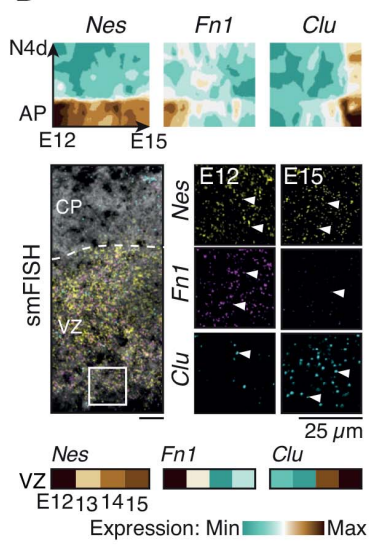

C 1. Cluster analysis of highly 2. Match remaining genes variable transcriptional maps with corresponding

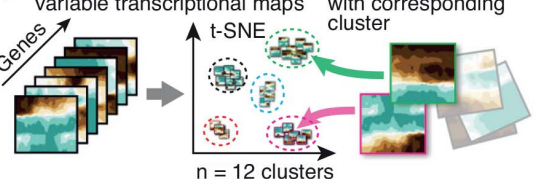

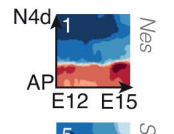
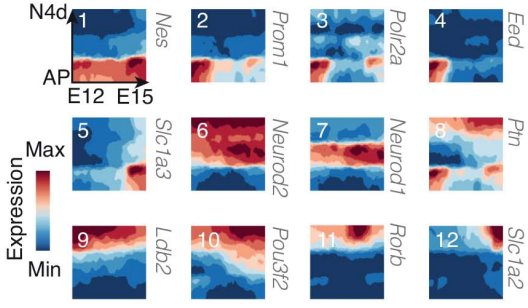

\section{D}

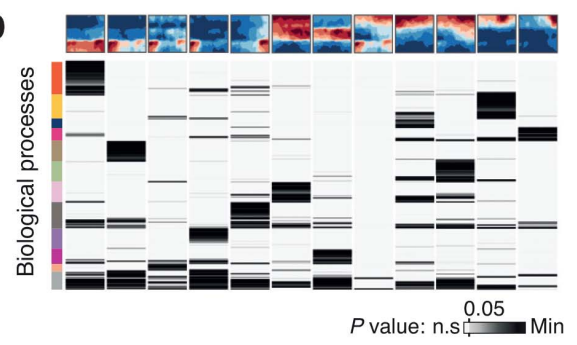

Keywords:

- Cell cycle / MAPK

Receptor / synaptic / G-proteins

- Cytoskeleton / neurotransmission

- Synapse / apoptosis

- DNA packaging / cell cycle

Axon and synapse

RNA processing / c. migration

- Metabolism

- MAPK / JNK

- TCR-like

DNA repair / transcription temporal progression of AP identity, we generated a cortex-specific knockout mouse for Eed, a regulatory subunit of PRC2 (Eed cKO mice). Loss of Eed eliminated PRC2's signature methylation mark in APs, consistent with a loss-of-function phenotype (Fig. 6B). As previously reported in the absence of another subunit of PRC2 (41), the thickness of the neocortex was strongly decreased in Eed cKO mice, consistent with a rapid temporal progression of APs resulting in a shortened neurogenic period. This possibility was supported by our observation that decreases in VZ thickness and increases in cell cycle exit unfolded precociously in Eed cKO cortex compared to wild-type mice (Fig. 6, C and D). To directly investigate whether the neurogenic competence of $\mathrm{Eed}^{-/-}$ APs was accelerated, we fate-mapped E14-born neurons using FT pulse-labeling. In contrast to wild-type cortex, in which essentially only L4 neurons are generated at this time, neurons with laminar and molecular features of normally laterborn L2/3 neurons were being generated in the
Eed mutant (Fig. 6E). Together, these results reveal that PRC2 exerts a fine-grained control over the temporal unfolding of successive states in APs and their daughter neurons (Fig. 6F).

\section{Discussion}

Together, our findings identify a combinatorial process in which cell type-specific neuronal identity emerges from the apposition of generic differentiation programs onto embryonic age-dependent, AP-derived transcriptional states. In this scenario, neuronal differentiation essentially corresponds to the implementation of programs coding for generic neuronal features (e.g., neurites, neurotransmission) onto temporally defined initial transcriptional states. This process is reminiscent of how neuron diversity is generated in evolutionarily older brain regions such as the subpallium or spinal cord $(7,24,42,43)$. In the subpallium, recent data in fact directly support largely conserved differentiation paths for distinct subtypes of inhibitory interneurons $(7,24,43,44)$, with the difference that in these regions, distinctions in initial neuronal states reflect a predominantly spatial rather than temporal distribution of molecularly distinct progenitors. These two modes of generating neuron diversity (i.e., temporal or spatial patterning) are in fact related, because the temporally coordinated expression of genes across adjacent cells is required to delineate molecularly defined areas. In evolutionary terms, temporal patterning may have been selected as the primary mode of neuron production within neocortical areas because it allows the generation of a large spectrum of cell types at low spatial cost.

In the course of corticogenesis, APs progressively become more exteroceptive. This suggests that extrinsic factors modulate neurogenesis late in development. Consistent with an increased receptivity of APs to their environment, their transcriptional identity shifts toward that of their neuronal progeny. This progressive acquisition of neuronal transcriptional features could 

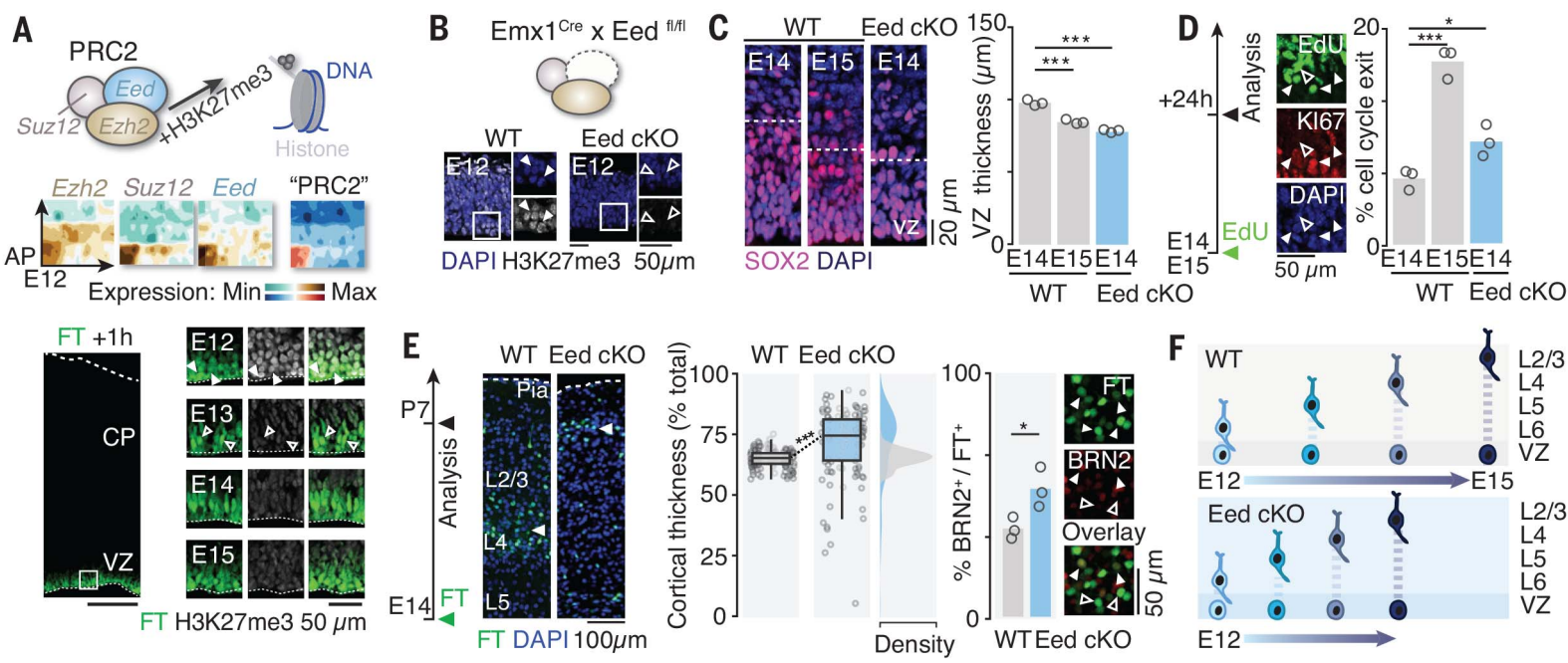

Fig. 6. PRC2 regulates the progression of AP temporal identity. (A) Top: Expression of the main subunits of the PRC2 complex is restricted to early APs. Bottom: The methylation mark of PRC2 has a corresponding temporal pattern. Dotted line indicates pial surface. (B) PRC2 function is disrupted in Eed cKO cortex, as shown by loss of the signature H3K27me3 mark.

(C) VZ thickness is precociously decreased in Eed cKO cortex. (D) Cell cycle exit is precociously decreased in Eed cKO cortex. (E) The laminar position and molecular identity of E14-born, FT pulse-labeled neurons in Eed cKO cortex is shifted toward that of normally later-born neurons. (F) Schematic summary of the findings. cKO, conditional knockout; DAPI, 4',6-diamidino-2phenylindole; PRC2, Polycomb repressive complex 2; WT, wild type. ${ }^{*} P<0.05$, ${ }^{* *} P<0.0001$ (Student $t$ test). reflect a decreased efficiency of the cyclic removal and insertion of epigenetic marks upon repetitive cell divisions, or the permeation of daughter neuron RNA or proteins into the mother cell.

Transmission of temporal birthmarks from mother to daughter cell may occur via conserved local- or large-scale chromatin features, as suggested by the critical role of epigenetic regulation in temporal patterning identified here. In addition, the passive transmission of cytoplasmic RNA and posttranscriptional events may also be involved $(17,45-48)$. This temporal birthmark fades with differentiation as environmental factors come into play. This process is particularly striking in developing L4 neurons, which are the main gateway for sensory input to the cortex, and whose final identity is sculpted by thalamocortical inputs $(3,37)$. These environmentdependent processes may occur in an areaspecific manner and may account for the recently reported molecular diversity in corresponding adult neuronal types across cortical areas (10). It will thus be interesting in future studies to understand how these environmental factors complement and eventually override earlier transcriptional processes, culminating in the generation of the full complement of cells required for functional cortical circuits.

\section{REFERENCES AND NOTES}

1. N. Gaspard et al., An intrinsic mechanism of corticogenesis from embryonic stem cells. Nature 455, 351-357 (2008) doi: 10.1038/nature07287; pmid: 18716623

2. M. Okamoto et al., Cell-cycle-independent transitions in temporal identity of mammalian neural progenitor cells. Nat. Commun. 7, 11349 (2016). doi: 10.1038/ncomms11349; pmid: 27094546

3. D. Jabaudon, Fate and freedom in developing neocortical circuits. Nat. Commun. 8, 16042 (2017). doi: 10.1038/ ncomms16042; pmid: 28671189
4. M. Kohwi, C. Q. Doe, Temporal fate specification and neural progenitor competence during development. Nat. Rev. Neurosci. 14, 823-838 (2013). doi: 10.1038/nrn3618; pmid: 24400340

5. A. Zeisel et al., Cell types in the mouse cortex and hippocampus revealed by single-cell RNA-seq. Science 347, 1138-1142 (2015). doi: 10.1126/science.aaa1934; pmid: 25700174

6. B. Tasic et al., Adult mouse cortical cell taxonomy revealed by single cell transcriptomics. Nat. Neurosci. 19, 335-346 (2016). doi: 10.1038/nn.4216; pmid: 26727548

7. T. J. Nowakowski et al., Spatiotemporal gene expression trajectories reveal developmental hierarchies of the human cortex. Science 358, 1318-1323 (2017). doi: 10.1126/ science.aap8809; pmid: 29217575

8. A. Saunders et al., Molecular diversity and specializations among the cells of the adult mouse brain. Cell 174, 1015-1030 e16 (2018). doi: 10.1016/j.cell.2018.07.028; pmid: 30096299

9. A. Zeisel et al., Molecular architecture of the mouse nervous system. Cell 174, 999-1014.e22 (2018). doi: 10.1016/ j.cell.2018.06.021; pmid: 30096314

10. B. Tasic et al., Shared and distinct transcriptomic cell types across neocortical areas. Nature 563, 72-78 (2018). doi: 10.1038/s41586-018-0654-5; pmid: 30382198

11. J. Kageyama, D. Wollny, B. Treutlein, J. G. Camp, ShinyCortex: Exploring single-cell transcriptome data from the developing human cortex. Front. Neurosci. 12, 315 (2018). doi: 10.3389/ fnins.2018.00315; pmid: 29867326

12. L. Telley et al., Sequential transcriptional waves direct the differentiation of newborn neurons in the mouse neocortex. Science 351, 1443-1446 (2016). doi: 10.1126/science.aad8361; pmid: 26940868

13. S. Govindan, P. Oberst, D. Jabaudon, In vivo pulse labeling of isochronic cohorts of cells in the central nervous system using FlashTag. Nat. Protoc. 13, 2297-2311 (2018). doi: 10.1038/ s41596-018-0038-1; pmid: 30258174

14. C. Trapnell et al.. The dynamics and regulators of cell fate decisions are revealed by pseudotemporal ordering of single cells. Nat. Biotechnol. 32, 381-386 (2014). doi: 10.1038/ nbt.2859; pmid: 24658644

15. S. A. Yuzwa et al., Developmental emergence of adult neural stem cells as revealed by single-cell transcriptional profiling. Cell Rep. 21. 3970-3986 (2017). doi: 10.1016/ j.celrep.2017.12.017; pmid: 29281841

16. E. Azim, S. J. Shnider, G. Y. Cederquist, U. S. Sohur, J. D. Macklis, Lmo4 and Clim1 progressively delineate cortical projection neuron subtypes during development. Cereb. Cortex 19 (suppl. 1), i62-i69 (2009). doi: 10.1093/cercor/bhp030 pmid: 19366868
17. S. K. Zahr et al., A translational repression complex in developing mammalian neural stem cells that regulates neuronal specification. Neuron 97, 520-537.e6 (2018). doi: 10.1016/j.neuron.2017.12.045; pmid: 29395907

18. O. Marín, Cellular and molecular mechanisms controlling the migration of neocortical interneurons. Eur. J. Neurosci. 38 2019-2029 (2013). doi: 10.1111/ejn.12225; pmid: 23651101

19. B. Wamsley, G. Fishell, Genetic and activity-dependent mechanisms underlying interneuron diversity. Nat. Rev. Neurosci. 18, 299-309 (2017). doi: 10.1038/nrn.2017.30; pmid: 28381833

20. B. Nadarajah, J. G. Parnavelas, Modes of neuronal migration in the developing cerebral cortex. Nat. Rev. Neurosci. 3 , 423-432 (2002). doi: 10.1038/nrn845; pmid: 12042877

21. Y. Yokota et al., Radial glial dependent and independent dynamics of interneuronal migration in the developing cerebral cortex. PLOS ONE 2, e794 (2007). doi: 10.1371/journal. pone.0000794; pmid: 17726524

22. J. D. Cahoy et al., A transcriptome database for astrocytes, neurons, and oligodendrocytes: A new resource for understanding brain development and function. J. Neurosci. 28, 264-278 (2008). doi: 10.1523/JNEUROSCI.4178-07.2008; pmid: 18171944

23. S. Minocha et al., Nkx2.1 regulates the generation of telencephalic astrocytes during embryonic development. Sci. Rep. 7, 43093 (2017). doi: 10.1038/srep43093; pmid: 28266561

24. C. Mayer et al., Developmental diversification of cortical inhibitory interneurons. Nature 555, 457-462 (2018). doi: 10.1038/nature25999; pmid: 29513653

25. J. Nishino, I. Kim, K. Chada, S. J. Morrison, Hmga2 promotes neural stem cell self-renewal in young but not old mice by reducing p16Ink4a and p19Arf Expression. Cell 135, 227-239 (2008). doi: 10.1016/j.cell.2008.09.017; pmid: 18957199

26. Y. Shimono, H. Murakami, Y. Hasegawa, M. Takahashi, RET finger protein is a transcriptional repressor and interacts with enhancer of polycomb that has dual transcriptional functions. J. Biol. Chem. 275, 39411-39419 (2000). doi: 10.1074/ jbc.M006585200; pmid: 10976108

27. C. L. Cunningham, V. Martínez-Cerdeño, S. C. Noctor, Microglia regulate the number of neural precursor cells in the developing cerebral cortex. J. Neurosci. 33, 4216-4233 (2013). doi: 10.1523/ JNEUROSCI.3441-12.2013; pmid: 23467340

28. I. Vitali et al., Progenitor Hyperpolarization Regulates the Sequential Generation of Neuronal Subtypes in the Developing Neocortex. Cell 174, 1264-1276.e15 (2018). doi: 10.1016/ j.cell.2018.06.036; pmid: 30057116

29. T. A. Weissman, P. A. Riquelme, L. Ivic, A. C. Flint, A. R. Kriegstein, Calcium waves propagate through radial glia 
cells and modulate proliferation in the developing neocortex. Neuron 43, 647-661 (2004). doi: 10.1016/j.neuron.2004.08.015; pmid: 15339647

30. M. Knobloch et al., Metabolic control of adult neural stem cell activity by Fasn-dependent lipogenesis. Nature 493 . 226-230 (2013). doi: 10.1038/naturel1689; pmid: 23201681

31. T. Takahashi, R. S. Nowakowski, V. S. Caviness Jr., The cell cycle of the pseudostratified ventricular epithelium of the embryonic murine cerebral wall. J. Neurosci. 15, 6046-6057 (1995). doi: 10.1523/JNEUROSCI.15-09-06046.1995; pmid: 7666188

32. B. Martynoga, H. Morrison, D. J. Price, J. O. Mason, Foxg1 is required for specification of ventral telencephalon and region-specific regulation of dorsal telencephalic precursor proliferation and apoptosis. Dev. Biol. 283, 113-127 (2005). doi: 10.1016/j.ydbio.2005.04.005; pmid: 15893304

33. Y. Sela, N. Molotski, S. Golan, J. Itskovitz-Eldor, Y. Soen, Human embryonic stem cells exhibit increased propensity to differentiate during the G1 phase prior to phosphorylation of retinoblastoma protein. Stem Cells 30, 1097-1108 (2012). doi: 10.1002/stem.1078; pmid: 22415928

34. A. Soufi, S. Dalton, Cycling through developmental decisions: How cell cycle dynamics control pluripotency, differentiation and reprogramming. Development 143, 4301-4311 (2016). doi: 10.1242/dev.142075; pmid: 27899507

35. D. Jabaudon et al., Inhibition of uptake unmasks rapid extracellular turnover of glutamate of nonvesicular origin Proc. Natl. Acad. Sci. U.S.A. 96, 8733-8738 (1999). doi: 10.1073/pnas.96.15.8733; pmid: 10411944

36. M. Eiraku et al., Self-organized formation of polarized cortical tissues from ESCs and its active manipulation by extrinsic signals. Cell Stem Cell 3, 519-532 (2008). doi: 10.1016/ j.stem.2008.09.002; pmid: 18983967

37. G. Pouchelon et al., Modality-specific thalamocortical inputs instruct the identity of postsynaptic L4 neurons. Nature 511 , 471-474 (2014). doi: 10.1038/nature13390; pmid: 24828045

38. D. Jabaudon, S. J. Shnider, D. J. Tischfield, M. J. Galazo, J. D. Macklis, ROR $\beta$ induces barrel-like neuronal clusters in the developing neocortex. Cereb. Cortex 22, 996-1006 (2012). doi: 10.1093/cercor/bhr182; pmid: 21799210
39. E. Klingler et al., A translaminar genetic logic for the circuit identity of intracortically projecting neurons. Curr. Biol. 29, 332-339.e5 (2019). doi: 10.1016/j.cub.2018.11.071; pmid: 30639110

40. R. Margueron, D. Reinberg, The Polycomb complex PRC2 and its mark in life. Nature 469, 343-349 (2011). doi: 10.1038/ nature09784; pmid: 21248841

41. J. D. Pereira et al., Ezh2, the histone methyltransferase of PRC2, regulates the balance between self-renewal and differentiation in the cerebral cortex. Proc. Natl. Acad. Sci. U.S.A. 107, 15957-15962 (2010). doi: 10.1073/pnas.1002530107; pmid: 20798045

42. J. S. Dasen, Transcriptional networks in the early development of sensory-motor circuits. Curr. Top. Dev. Biol. 87, 119-148 (2009). doi: 10.1016/S0070-2153(09)01204-6; pmid: 19427518

43. D. Mi et al., Early emergence of cortical interneuron diversity in the mouse embryo. Science 360, 81-85 (2018). doi: 10.1126/ science.aar6821; pmid: 29472441

44. L. Telley, D. Jabaudon, A mixed model of neuronal diversity. Nature 555, 452-454 (2018). doi: 10.1038/d41586-018-02539-4; pmid: 29565398

45. K.-J. Yoon et al., Temporal control of mammalian cortical neurogenesis by m6a methylation. Cell 171, 877-889.e17 (2017). doi: 10.1016/j.cell.2017.09.003; pmid: 28965759

46. K.-J. Yoon, C. Vissers, G.-L. Ming, H. Song, Epigenetics and epitranscriptomics in temporal patterning of cortical neural progenitor competence. J. Cell Biol. 217, 1901-1914 (2018). doi: 10.1083/jcb.201802117; pmid: 29666150

47. M. Z. Ozair et al., hPSC Modeling reveals that fate selection of cortical deep projection neurons occurs in the subplate. Cell Stem Cell 23, 60-73.e6 (2018). doi: 10.1016/ j.stem.2018.05.024; pmid: 29937203

48. T. J. Nowakowski et al., Regulation of cell-type-specific transcriptomes by microRNA networks during human brain development. Nat. Neurosci. 21, 1784-1792 (2018). doi: 10.1038/s41593-018-0265-3; pmid: 30455455

\section{ACKNOWLEDGMENTS}

We thank A. Benoit and the Genomics Platform and FACS Facility of the University of Geneva for technical assistance, and $\mathrm{H}$. Wu,
E. Azim, O. Raineteau, D. Silver, and the members of the Jabaudon laboratory for comments on the manuscript. Illustration in panel 3D: www.lagraphisterie.fr. Funding: Work in the Jabaudon laboratory is supported by the Swiss National Science Foundation and the Carigest Foundation. The Nguyen laboratory is funded by the FRS-FNRS (EOS 0019118F-RG36: CDR J.0028.18; PDR T.0073.15), the Fonds Léon Fredericq, the Fondation Médicale Reine Elisabeth, and the Fondation Simone et Pierre Clerdent. Work in the Hippenmeyer laboratory is supported by IST Austria institutional funds and the European Research Council (ERC) under the European Union's Horizon 2020 Research and Innovation Program (grant agreement 725780 LinPro). G.A. is a doctoral student from the FRS-FNRS and is supported by the Fonds Léon Fredericq. Also supported by the Fondation privée des HUG (D.J. and A.D.); the Swiss National Science Foundation and the National Center of Competence in Research (NCCR) Synapsy (A.D.); and FWF Hertha Firnberg Program grant T 1031-BBL (N.A.) Author contributions: L.T. and G.A. performed the experiments with the help of P.O. and I.V.; L.T., G.A., and J.P. performed the bioinformatic analysis; S.F. performed the electrophysiology experiments; C.C. performed the smFISH experiment; G.B. performed the in vitro experiments; N.A. performed the Eed cKO-related experiments; and G.A., D.J., and L.T. wrote the manuscript with the help of A.D., L.N., and S.H. Competing interests: None. Data and materials availability: All annotated data are available at the GEO database (accession number GSE118953) or in the open access website (http://genebrowser.unige.ch/telagirdon/). All other data (beyond GEO or GeneBrowser) are in the main paper or the supplement.

\section{SUPPLEMENTARY MATERIALS}

science.sciencemag.org/content/364/6440/eaav2522/suppI/DC1 Materials and Methods

Figs. S1 to S10

Tables S1 and S2

References (49-56)

Data S1 to S8

5 September 2018; accepted 4 April 2019

10.1126/science.aav2522 


\section{Science}

\section{Temporal patterning of apical progenitors and their daughter neurons in the developing neocortex}

L. Telley, G. Agirman, J. Prados, N. Amberg, S. Fièvre, P. Oberst, G. Bartolini, I. Vitali, C. Cadilhac, S. Hippenmeyer, L. Nguyen, A. Dayer and D. Jabaudon

Science 364 (6440), eaav2522.

DOI: 10.1126/science.aav2522

\section{Origins of neuronal diversity}

Although the main task of a neuroprogenitor is to produce more cells, it may not always produce the same cells. Some progenitors produce different daughter neurons as an embryo develops. Concurrently, these daughter neurons are also transitioning through states toward maturation. Telley et al. used single-cell RNA sequencing to survey the transcriptional identity of cells early in mouse brain development. As a neuroprogenitor transitioned to new states, it produced daughter neurons that reflect those new states. The neuron's own postmitotic differentiation program is apparently overlaid onto these parentally supplied programs, driving emergence of specialized neuronal cell types in the neocortex.

Science, this issue p. eaav2522

ARTICLE TOOLS

SUPPLEMENTARY MATERIALS

REFERENCES

PERMISSIONS http://science.sciencemag.org/content/364/6440/eaav2522

http://science.sciencemag.org/content/suppl/2019/05/08/364.6440.eaav2522.DC1

This article cites 56 articles, 16 of which you can access for free http://science.sciencemag.org/content/364/6440/eaav2522\#BIBL

http://www.sciencemag.org/help/reprints-and-permissions

Use of this article is subject to the Terms of Service

Science (print ISSN 0036-8075; online ISSN 1095-9203) is published by the American Association for the Advancement of Science, 1200 New York Avenue NW, Washington, DC 20005. 2017 () The Authors, some rights reserved; exclusive licensee American Association for the Advancement of Science. No claim to original U.S. Government Works. The title Science is a registered trademark of AAAS. 